\title{
Building the Program Parallelization System Based on a Very Wide Spectrum Program Transformation System
}

\author{
Alexander Alexeyevich Bukatov \\ Rostov State University, Computer Center, 200/1, build. 2, Stachki Ave., \\ 344090 Rostov-on-Don, Russia \\ baa@rsu.ru \\ http://uginfo.rsu.ru
}

\begin{abstract}
An approach to build an automated Program Parallelization System based on a multipurpose very wide spectrum program transformation system is discussed. The main advantage of the proposed approach is a nonprocedural way in which the parallelizing transformations are described in the Program Transformation System. It allows doing any required customization of the Program Parallelization System for a new parallel computer platform or new parallelization techniques. General organization of the proposed Multipurpose Program Transformation System is discussed, some examples of nonprocedural representations of parallelizing transformations are analyzed and comparison with related works is provided.
\end{abstract}

\section{Introduction}

Automated parallelization tools provide the means for porting legacy sequential programs to parallel computer platforms and using sequential programming languages to develop new programs for parallel computers. The program parallelization techniques were being widely investigated in the last decades. For some representative classes of parallel computers with shared memory, vector and SMP architecture computers included, effective parallelization techniques had been developed. Basic vendors of parallel computers with such architectures have provided the parallelizing compilers for these types of computers. Nevertheless, for the parallel computer architectures with distributed memory (DM) such as MP, cluster or NUMA architectures more or less effective parallelization techniques are still poorly developed and numerous investigations in this direction are being leaded. Some new techniques of program parallelization for DM computers are being introduced from time to time. Therefore there is a need to change each time the implementation of experimental program parallelization tools that have been developed for DM computers to incorporate the new techniques. Building an automated program parallelization tools based on nonprocedural description of parallelizing transformations may dramatically simplify the evolutionary development of such tools.

The other reason for building the program parallelization tools around the nonprocedural description or parallelizing transformations arises from the practical need how 
to "teach" the existing parallelization tools to apply new parallelizing transformation developed by skilled users of such tools. The application domain specific parallelization methods that can be developed by skilled application programmers for parallelizing some high-level multi-component program structures is of special interest.

This paper discusses the way of building an extendable Automated Program Parallelization System (APPS) based on a very wide spectrum Multipurpose Program Transformation System (MPTS) [1,2]. The next section of the paper discusses the main features of the MPTS and of underlying program transformation language. In Section 3 the general organization of the MPTS and APPS is observed. In Section 4 two examples of parallelizing program transformations are discussed. In Conclusion general assessment of this paper and comparison of discussed results with related investigations are provided.

\section{Main Features of the Multipurpose Program Transformation System and Language}

The Multipurpose Program Transformation System (MPTS) like most others program transformation systems $[3,4]$ is based on a nonprocedural representation of program transformations in the form of Schematic Transformation Rules.

The basic notion used in the area of schematic program transformation is a program scheme [3]. A program scheme is a representation of some class of similar programs or similar program structures (statements). It originates from the concrete program or from the program structure by parameterization: some components of the program (of the program structure) are replaced by the parameters named.

Schematic transformation rule (STR) is the mapping (probably a partial one) from the space of program schemes to the same space. Usually [3,4] this mapping is recorded in the following form:

\section{input-pattern \& enabling-condition $\Rightarrow$ output-pattern}

where the input-pattern is the scheme of the transformed structure and the outputpattern is the scheme of transformation result. The enabling-condition, which usually depends on the input-pattern parameters, restricts the STR applicability.

The STRs can be applied to any program for its transformation. The STR application includes the following stages. First, all the structures (statements) of the transformed program matching the input pattern are found. Simultaneously for each matching the values of input pattern schema parameters are determined (unified). Then for each matching the enabling condition is evaluated. If for some source structure matched to the input pattern this value is 'TRUE', then this source structure is replaced by the structure produced from the output pattern by substituting the unified parameter values.

A single STR or some set of STRs can be applied once or repeatedly "by exhaustion", i.e. until no matches of its input pattern are found. The order of the STRs application can be specified in a program transformation scenario expressed in an appro- 
priate program transformation language. This provides the means to represent both the STRs and the program transformation scenarios.

The STR form discussed above is used in all known program transformation systems (PTS) [3,4]. It provides the way for nonprocedural representation of rather simple program transformations and cannot cover all the needs for implementation of the well-known program parallelization and vectorization techniques (see [5-7]). Local transformations can only be represented in the STR form. But not all program parallelization transformations are the local ones. The typical example of a non-local transformation is the loop restructuring because usually the statements that declare and initialize the loop variables are involved in this transformation. Moreover not all the local program transformations can be expressed in a natural way in the STR form. For example, to restructure some loop statement to a parallelized program structure some Diophantine equations should be solved. It's obvious enough that the algorithm used for solution of equations cannot be expressed efficiently in the form of the program rewriting rules.

So the expressive power of the traditional schematic program transformation languages and systems is not good enough to be used for the implementation of program parallelization systems. The MPTS suggested in [1,2] extends the traditional program transformation languages in two main directions. First, sophisticated compound multicomponent patterns are introduced. And second, some functional capabilities are introduced into a program transformation language. Additionally, some interactive features are introduced to the MPTS.

Let's consider the Multipurpose Program Transformation Language (MPTL) and System (MPTS). The core construction of the MPTL is the declaration of the Program Transformation Rule (PTR) that is of the following form:

rule rule-name (rule-parameters-list) [var variable-declarations-list ; ] compound-input-pattern

$\& \&$ enabling-condition $=>$ compound-output-pattern

end

Here and everywhere in this paper the names of non-terminal constructions are printed in the italic font and the square brackets are used to enclose optional constructions.

In the variable declarations list rule parameters as well as input and output pattern parameters are declared. The declaration of each parameter includes the name of this parameter syntax type (the name of the corresponding non-terminal symbol of the transformed program grammar). It means that only the string derivable from the defined non-terminal symbol can be matched to this parameter. It provides the basis for the efficient implementation of the matching process.

Now let's consider the most important parts of the PTR declaration, the compound input and output patterns. Each of these patterns can be composed of several pattern or procedural terms. The definition of compound input pattern, for example, has the following syntax:

compound-input-pattern ::= pop-term $\{\&$ pop-term $\}$

pop-term $::=$ pattern-term $\mid$ procedural-term 
Here and further in the paper the '::=' symbol is used to separate the left and right parts of the syntax rule; the figure brackets ' $\{$ ' and ' $\}$ ' are used to enclose a construction that can be repeated 0 or more times; the ' $I$ ' symbol separates alternatives of the syntax rule and the ' ' symbol ends the syntax rule.

The first term of the input pattern is usually the pattern-term. It provides the schema of the root (or, in other words, the 'anchor') component of a compound program structure. Each of the subsequent pattern terms is as a rule connected with one of the previous terms by a semantic relation or by common parameters. The procedural terms are used to transform their input parameters to the output ones when the algorithm of the required transformation is rather sophisticated. The simplified syntax of the pattern term has the following form:

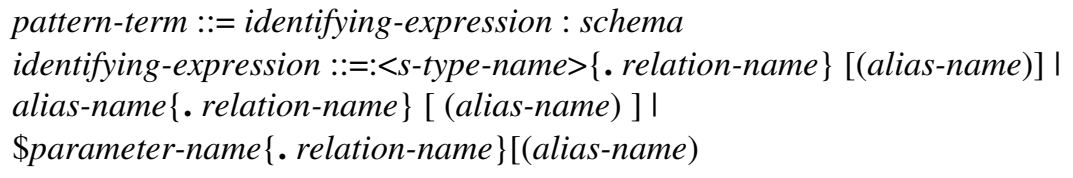

The form of identifying expressions is mostly based on a semantic model of the transformed program that is used in the MPTS. According to this model the transformed program is treated as a hierarchical structure of the syntax objects connected by the binary semantic relations such as "declared in". The hierarchical links are also considered as the named binary relations. The whole program structure is considered to be a directed graph whose nodes are the syntax objects and edges are the named relations.

The simplest case of an identifying expression is the syntax type name (s-type-name). In this case all the syntax objects of s-type-name type are compared with the schema during the matching process. The fact that the syntax type of the matched object is known before matching simplifies the matching process dramatically and provides the means for its efficient implementation. A more complex form of identifying expressions is based on the principles of a navigational query language of special object-oriented database [8,9]. In this case the identifying expression has the form of the dot separated chain of relation names that defines some path in the graph program structure leading from the named object to the object being identified.

The alias name optionally provided in the identifying expression is used to refer to the matched object from others pattern terms. In the PTR output pattern the three following additional forms of identifying expression of the pattern terms can also be used: before(alias-name), instead-of(alias-name) and after(alias-name). Note that the simple alias-name form is equal to instead-of(alias-name) when used in the out pattern.

The procedural-pattern is the way to capture procedural transformation steps into schematic transformation process. The simplest form of the procedural term is an external procedure call. This procedure can be implemented in some traditional programming language (the interface to $C$ language is supported in the MPTS).

The last PTR component, which has not been discussed yet, is the enabling condition. It is the logical expression that depends on the input pattern parameters. This expression can include some external predicates (i.e. functions that return Boolean value) as operands. One of such predicates predefined in the MPTS is used to intro- 
duce interactivity to a transformation process. It asks a programmer a parameterized question and transforms his 'yes' or 'no' reply to the Boolean 'TRUE' or 'FALSE' values respectively that are returned to the transformation engine. A more complex form of an enabling condition is beyond of the scope of this paper.

The MPTL features discussed in this section can sufficiently widen the area of the program transformation approach applicability, so that MPTL can be used to represent rather complex parallelizing transformations. Almost all of the discussed extensions of traditional program transformation languages have been introduced by us. Let us note, that in works of V.L. Winter, dealing with HATS system [10,11], the pattern schemas and parameters are also marked by syntax types of corresponding syntax objects. This, as it has been mentioned above, provides the means for efficient implementation of the matching process. Winter has also tried to overcome the gap between the local and global transformations by introducing special wildcard symbol '*', which can be matched with an arbitrary string. But this attempt is not considered to be successful one because a wildcard symbol cannot be implemented efficiently and it does not really provide means to represent complex semantic structures composed of distributed components.

\section{General Organization of the Multipurpose Program Transformation and Automated Program Parallelization Systems}

The transformed programs are represented in the MPTS in the internal form and are stored in the object database (ODB) [8-9]. The primary form of the internal representation of the transformed programs is the Abstract Syntax Tree (AST) extended by additional semantic links (relations), which connect the AST nodes. On the bases of the extended AST some additional secondary representations such as the Control Flow Graph (CFG) and the Data Dependency Graph (DDG) are produced. These secondary representations are used to implement efficiently different predicates intended for application in PTR enabling conditions.

The MPTS is composed of the tools (subsystems), which provide the following main functions:

- conversion of source programs to their internal presentation stored in the ODB and vice versa;

- support the PTRs and PTSs (program transformation scenarios) creation an modification (the aggregate of PTRs and PTSs play the role of the APPS's knowledge base);

- application of the PTRs and PTSs to the transformed program to produce the transformation result.

The tools, converting source programs to their internal presentation and vice versa (below, the program conversation tools) can be customized to the syntax of programming languages that are used to represent source sequential programs and target parallel programs, to be created as the result of the parallelization. In fact a combined 
language, containing constructions of both sequential and parallel language is constructed and its syntax in the form of an L-attributed grammar [12] is defined. Then the definition of this grammar $\boldsymbol{G}$ is entered into the MPTS. The syntax rules of the grammar $\boldsymbol{G}$ are compiled to C-procedures, which make the transformation of the source program to an attributed AST and vice versa. On the AST basis the required secondary presentations are produced such as CFG and DDG. Let us note that all the PTRs when applied to the transformed programs actually operate on the AST level that is very close to the source program level. Secondary representations are reconstructed by the MPTS after each AST transformation. The techniques of such reconstruction are discussed in the paper [13] written under our guidance.

Tools intended for the PTRs and PTSs creation and modification (below, the PTR creation tools) do compile the transformation programs expressed in the form of PTSs and PTRs to C-procedures that perform the corresponding transformation. Such approach provides the simple way to implement the MPTL functional (procedural) capabilities including procedural terms of the PTR patterns and "embedded" predicates used in enabling conditions. The PTR creation tools also provide the check of PTR structural correctness. Each pattern term is checked for consistency with the $\boldsymbol{G}$ grammar.

The transformation engine provides the PTSs and/or PTRs (MPTL programs) application to the transformed program. It is the main part of the MPTS. A MPTL program can be applied to the entire program, which is to be transformed, or to some identified part of this program. Both the interactive interface and the application programmer interface (API) to the transformation engine are provided by the MPTS. The API interface is provided through the apply function. Note, if the transformation engine API is provided, any traditional programming language can be used as means to code program transformation scenarios.

The automated program parallelization system (APPS) is built on the MPTS basis in the following way. The set of "predefined" predicates and functions (procedures) intended for application in the PTR's enabling conditions and procedural pattern terms is implemented in the $\mathrm{C}$ programming language. This set forms the procedural part of the APPS knowledge base. The nonprocedural part of the APPS knowledge base is represented in the PTRs form. This approach was used in paper [14] to implement classical parallelization techniques of R. Allen and K. Kennedy [5] in the form of the APPS knowledge base.

\section{Examples of the Nonprocedural Representation of Parallelizing Transformations}

Two examples are provided in this section. The first example is simplified to be expressed mainly with the MPTL features discussed in section 2. The second example is rather complicated and demonstrates some additional features of the MPTL.

Let's consider the following program structure that is coded in Pascal like language as a first example: 
$\mathrm{s}:$ real; . . . a: real array of $[1 . . \mathrm{n}] ; \ldots$

$\mathrm{s}:=0 ; \ldots$ for $\mathrm{I}:=1$ to $\mathrm{n}$ do $\mathrm{s}:=\mathrm{s}+\mathrm{a}[\mathrm{i}]$;

The loop, contained in this structure, can be replaced by two consecutive loops. The first one can be run in parallel where as the second one is similar to the source loop but involves $\mathrm{n} / 2$ (if $\mathrm{n}$ is even) iterations. The following PTR defines the transformation discussed.



In this example the input pattern of the sum_loop_tr PTR consists of three pattern terms. The identifying expression of the second term defines the path from the syntax construction matched with \$a parameter to the structure that should be matched with the second pattern term. The output pattern consists of two procedural terms and two pattern terms. The enabling condition is the conjunction of the three predicates.

Of course the sum_loop_tr PTR can be generalized. For example, the '+' operation in the loop statement as well as the ' 0 ' value in the assignment statement should be parameterized for any operation that is commutative and associative and for the 'NULL' value of this operation group. Moreover, the syntax of the $\$ \mathrm{~s}+\$ \mathrm{a}[\mathrm{\$ i}]$ expression should also be parameterized to be matched with expressions like $\operatorname{MIN}(\$ s, \$ a[\$ i])$. The MPTL language provides the mean of the required expression parameterization not discussed above. Pattern terms can be written in the attributed AST notation eliminating the difference between the infix and functional forms of the operation syntax.

As the second example we consider the PTR that implements the loop unrolling (see [5], for instance).

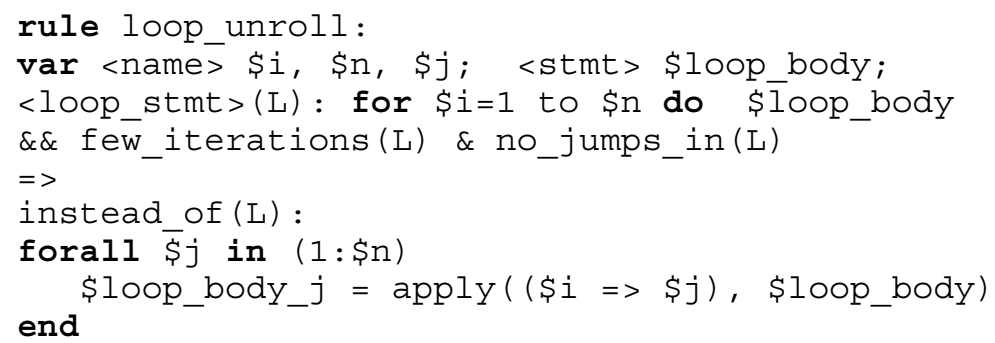


In this example, which in fact is also simplified, the loop unrolling transformation is represented.

Let's discuss two additional constructions of the MPTL that are used in the above example. The first one is the forall-in construction. This construction is used to produce sequentially the indexed samples of the embedded statement. In the MPTL there are some other constructions, which provide the means to handle and to explore the sets of the schemas. The second construction provides the way of the recursive call of the transformation engine. The apply function is called recursively in this example to apply the embedded anonymous transformation rule to the loop body.

There are some other features of the MPTL and MPTS that are not discussed in this paper. But the features discussed do demonstrate the expressive power of the MPTL, the potential efficiency of the MPTS implementation, and the applicability of the discussed tools for building the program transformation systems.

\section{Conclusion}

The main aim of this paper is to develop an approach to implement extendable program parallelization tools. This approach should provide the means for evolutionary extension of such tools to incorporate innovations of the underlying parallelization techniques. According to this aim the main achievements discussed in the paper are the following:

First, the principle has been suggested to build a program parallelization system on a basis of a schematic transformation system that allows representing parallelizing transformations in a nonprocedural form that is clearer for understanding and easier for modification than a procedural implementation of these transformations. As we know there are no other investigations that suggest any approach to the nonprocedural implementation of the parallelizing transformation and, partially, an approach based on a program transformation system.

Second, development of a schematic program transformation approach has been performed with the aim of increasing the expressive power of a program transformation language, which would be suitable for clear representation of complicated parallelizing transformations. The MPTL program transformation language providing new expressive features has been developed. The prototype implementation of the MPTS program transformation system based on the MPTL has been performed. The MPTS and MPTL do develop ideas of the traditional program transformation systems [3-4] in two main directions. The transformations of the compound multi-component program structures (the semantic structures composed of distributed components) can be defined in MPTL and applied by MPTS. And the procedural means (procedural terms and predicates) can be used where required. There are no other program transformation systems that provide the facilities mentioned above. The HATS system $[11,12]$ provides only some means to express non-local transformations (see the end of Section 2). But these means are restricted and are not powerful enough to represent com- 
plicated transformations. And there is no way to implement the wildcard feature efficiently.

And third, the MPTL application to represent of complex parallelizing transformations has been shown in the Section 4 and in the paper [14] written under our guidance.

Note that MPTS based PPS is, in fact, the meta-system that makes it possible to implement different parallelization techniques. In paper [14], the parallelizing transformations of R. Allen and K. Kennedy are represented in a non-procedural form. Other classical parallelization techniques [6,7], different extensions of these techniques (see, [15]) as well as parallelization techniques based on completely different approaches to parallelization can also be represented in this form. At present we are planning to implement the V-Ray parallelization technology [16] based on the MPTS.

Since we implement in nonprocedural form the parallelization techniques developed by other researchers the parallelizing power of our implementation is mainly determined by the underlying parallelization techniques. That is why we do not provide any test data based on PTS running on the Livermore Loops benchmark. By the similar reason no comparison of the PPS performance time parameters with other parallelization systems has been made.

\section{References}

1. Bukatov A.A. Development of Tools Intended for Creation of the Source Program Transformation Systems. Information Technologies, N 2, 1999, 22-25 (published in Russian).

2. Bukatov A. A. Development of Tools for Nonprocedural Implementation of Program Parallelizing Transformations. Proceedings of the Russian Conference "Fundamental and Application Aspects of Development of the Large Distributed Program Complexes", Abrau-Durso, MSU Publishing, 1998, 109-116 (published in Russian).

3. Partch H., Steinbruggen R. Program Transformation Systems. ACM Computing Surveys, 1983, v. 15, N 3, 199-236.

4. Visser E. A Survey of Rewriting Strategies in Program Transformation Systems. Electronic Notes in Theoretical Computer Science, v. 57 (2001), 35 p.

http://www.elsevier.nl/locate/entcs/volume57.html

5. Allen R., Kennedy K. Automatic Translation of FORTRAN Programs to Vector Form, 1987.

6. Bannerjee U. Dependence Analysis for Supercomputing. Kluver Academic Publishers, New York, 1988.

7. Wolfe M.J. Optimizing Supercompilers for Supercomputers. MIT Press, Cambridge, Mass, 1889.

8. Bukatov A.A., Zastavnoy D.A. High-level navigational language for querying complex data objects and its applications to CASE systems // Proc. of the 3rd Joint Conference on Knowledge-Based Software Engineering, Smolenice, Slovakia, 1998, pp. 103-107.

9. Zastavnoy D.A., Bukatov A.A. Representation of Complex Structures Extracted from Object Databases, and Access to their Components // In: Hruska T., Hasimoto M. (Eds) Knowledge-Based Software Engineering, Amsterdam: IOS Press, 2000, 93-100.

10. Winter V.L. An Overview of HATS: A Language Independent High Assurance Transformation System. Proc. of the IEEE Symposium on Application-Specific Systems and Software Engineering Technology (ASSET), March 24-27, 1999. 
11. Winter V.L. Program Transformation in HATS. Proceedings of the Software Transformation Systems Workshop, May 17, 1999.

12. Lewis P.M., Rosenkranz D.J., Stearns R.E. Attributed Translations. Journal of Computer and System Sciences, v. 9, N 3, 1974, 279-307.

13. Lugovoy V.V. Development of the Internal Representation Modification Techniques in the Program Parallelization CASE-system. Proceedings of the Conference "High Performance Computing and its Applications", Chernogolovka, Russia, 2000, 133-136 (published in Russian).

14. Zhegulo O. Representation of Knowledge on Program Parallelization Techniques in the Expert System Supporting Program Parallelization. Artificial Intelligence, the Journal of National Academy of Sciences of Ukraine, Institute of Artificial Intelligence, No 2001'3, Donetsk, 2001, 323-330 (published in Russian).

15. Pinter S.S., Pinter R.Y., Program Optimization and Parallelization Using Idioms. ACM Transactions on Programming Languages and Systems, 1994, vol. 16, N 3, 305-327.

16. Voevodin V.V., Voevodin Vl.V. V-Ray technology: a new approach to the old problem: Optimization of the TRFD Perfect Club Benchmark to CRAY Y-MP and CRAY T3D Supercomputers. Proceedings of the High Performance Computing Symposium'95, Phoenix, Arizona, USA, 1995, 380-385. 